Article

\title{
In Vitro and In Vivo Evaluation of Essential Oil from Artemisia absinthium L. Formulated in Nanocochleates against Cutaneous Leishmaniasis
}

\author{
Beatriz Tamargo ${ }^{1}$, Lianet Monzote ${ }^{2}$, Abel Piñón ${ }^{2}$, Laura Machín ${ }^{1}$, Marley García ${ }^{2}$, \\ Ramón Scull ${ }^{3}$ and William N. Setzer ${ }^{4, *}$ \\ 1 Department of Pharmacology, Institute of Pharmacy and Food, Havana University, Havana 10400, Cuba; \\ btamargo@infomed.sld.cu (B.T.); laura@ifal.uh.cu (L.M.) \\ 2 Parasitology Department, Institute of Tropical Medicine Pedro Kouri, Havana 10400, Cuba; \\ monzote@ipk.sld.cu (L.M.); abelpt@ipk.sld.cu (A.P.); marleygp@nauta.cu (M.G.) \\ 3 Department of Chemistry, Institute of Pharmacy and Food, Havana University, Havana 10400, Cuba; \\ rscull@ifal.ih.cu \\ 4 Department of Chemistry, University of Alabama in Huntsville, Huntsville, AL 35899, USA \\ * Correspondence: wsetzer@chemistry.uah.edu; Tel.: +1-256-824-6519
}

Academic Editor: Eleni Skaltsa

Received: 28 March 2017; Accepted: 5 June 2017; Published: 9 June 2017

\begin{abstract}
Background: Leishmaniasis is a zoonotic disease caused by protozoan parasites from Leishmania genus. Currently, there are no effective vaccines available and the available therapies are far from ideal. In particular, the development of new therapeutic strategies to reduce the infection caused by Leishmania amazonensis could be considered desirable. Different plant-derived products have demonstrated antileishmanial activity, including the essential oil (EO) from Artemisia absinthium L. (EO-Aa), Asteraceae. Methods: In the present study, the EO-Aa formulated in nanocochleates (EO-Aa-NC) was investigated in vitro against intracellular amastigotes of L. amazonensis and non-infected macrophages from BALB/c mice. In addition, the EO-Aa-NC was also evaluated in vivo against on experimental cutaneous leishmaniasis, which body weight, lesion progression, and parasite load were determined. Results: EO-Aa-NC displayed IC $_{50}$ values of $21.5 \pm 2.5 \mu \mathrm{g} / \mathrm{mL}$ and $27.7 \pm 5.6 \mu \mathrm{g} / \mathrm{mL}$ against intracellular amastigotes of L. amazonensis and non-infected peritoneal macrophage, respectively. In the animal model, the EO-Aa-NC $(30 \mathrm{mg} / \mathrm{kg} /$ intralesional route/every 4 days 4 times) showed no deaths or weight loss greater than 10\%. In parallel, the EO-Aa-NC suppressed the infection in the murine model by approximately $50 \%$, which was statistically superior $(p<0.05)$ than controls and mice treated with EO-Aa. In comparison with Glucantime ${ }^{\circledR}$, EO-Aa-NC inhibited the progression of infection as efficiently $(p>0.05)$ as administration of the reference drug. Conclusions: Encochleation of EO-Aa resulted in a stable, tolerable, and efficacious antileishmanial formulation, facilitating systemic delivery of $\mathrm{EO}$, with increased activity compared to administration of the free EO-Aa. This new formulation shows promising potential to future studies aimed at a new therapeutic strategy to treat leishmaniasis.
\end{abstract}

Keywords: Artemisia absinthium; essential oil; Leishmania amazonensis; cutaneous leishmaniasis; nanocochleate

\section{Introduction}

Leishmaniasis is a zoonotic disease caused by protozoan parasites from Leishmania genus (Trypanosomatidae), which is a transmitted by the bites of infected female sandflies. Currently, over 20 species of protozoan have been reported as pathogenic to humans [1]. This obligate intracellular parasite has a complex digenetic life cycle, which requires a susceptible vertebrate host, as well as 
a permissive insect vector, where they exist as amastigote or promastigote forms, respectively [2]. Leishmania is capable of developing a wide spectrum of diseases, including: cutaneous (CL), mucosal (ML), and visceral (VL) leishmaniasis; with symptoms ranging from cutaneous lesions that destroy the skin to visceral compromise of important organs, such as liver and spleen [3]. The disease presents high morbidity and mortality rates throughout the world, where around 350 million people from 98 countries are at risk of contracting the disease [4]. Currently, leishmaniasis diagnosis is performed by a combination of clinical, epidemiological, and laboratorial data, which can lead to several false-negatives, delaying early implementation of treatment and, thus, contribute to the maintenance of reservoirs, thereby preserving of parasitological cycles in their environment [5]. There are no effective vaccines currently available for any form of leishmaniasis, while the available therapies are far from ideal due to their toxicity, high costs, lack of efficacy, difficult access in certain areas, and emerging drug resistance [6]. Leishmaniasis is currently classified as one of the neglected tropical diseases [1].

In particular, $\mathrm{CL}$ is one of the most common leishmaniasis forms and one of the major public health and social problems in developing countries and throughout the world. Leishmania amazonensis constitutes one of etiologic agents of New World CL. This form of the disease can be associated with the dissemination of parasites due to an inefficient immune response. On the other hand, L. amazonensis can also be involved in diffuse cutaneous leishmaniasis (DCL) syndrome, which generally cannot be controlled with conventional treatments due to a specific allergy to parasite antigens [7]. Pentavalent antimonials have been the first choice for treatment while other drugs, such as amphotericin B, pentamidine and paromomycin, are used as a second option in resistant cases [8]. In general, the several side effects registered (chest pain, shortness of breath, nausea, vomiting, diarrhea, irregular heartbeat, lethargy), the high cost and/or the limit efficacy have decreased the clinical use of these compounds, in particular to control the DCL [9-11]. In this sense, the development of new chemotherapeutic agents to reduce the infection caused by L. amazonensis should be considered desirable.

Different plant-derived products have demonstrated antileishmanial activity, in the search of better effects and less toxicity [12,13]. Recently, the essential oil (EO) from Artemisia absinthium L. (EO-Aa), Asteraceae, was shown to be active on promastigotes and intracellular amastigotes and demonstrated disease control in a BALB/c mouse model of experimental cutaneous leishmaniasis [14]. As EOs are complex mixtures of various volatile components, promising approaches have been reported using nanoencapsulated EOs in drug delivery systems to decrease their volatility and improve their stability, water solubility, and effectiveness of EO-based formulations to maintain desirable therapeutic efficacy [15]. Therefore, we hypothesize that the Artemisia-oil formulated in nanocochleates (EO-Aa-NC) could contribute to stability of EO and increase the activity which, in the present study, the in vitro and in vivo antileishmanial activity of was investigated.

\section{Materials and Methods}

\subsection{Plant Material and Essential Oil from A. absinthium}

In the early morning of July 2001, the leaves of A. absinthium were collected from the Pharmacy and Foods Institute, University of Havana, Cuba. A voucher specimen number of ROIG-4640 was assigned and a specimen was deposited in the Experimental Station of Medicinal Plants "Dr. Juan Tomás Roig", Cuba. Leaves were manually crushed and the EO was obtained by hydrodistillation of the aerial parts of the plant using Clevenger type equipment and characterized by gas chromatographic-mass spectral (GC-MS) analysis as previously reported [14]. EO-Aa was hermetically sealed and stored in the Natural Product Collection of Institute of Tropical Medicine Pedro Kouri under standard conditions $\left(4{ }^{\circ} \mathrm{C}\right.$ and darkness). 


\subsection{Reference Drugs}

Glucantime ${ }^{\circledR}$ (GTM) from Rhône-Poulenc Rorer, Ciudad De Mexico, Mexico, was used as a reference drug, which was dissolved in sterile distilled water at a concentration of $30 \mathrm{mg} / \mathrm{mL}$.

\subsection{Nanocochleate Preparation}

To prepare nanocochleates with the EO-Aa and the aqueous extract of pericarp from fruits of S. saponaria [16], the dehydration-hydration process described by Gregoriadis and collaborators [17] and modified by Tamargo and collaborators [18] was used with purified phospholipids (Flps) from soy lecithin [19]. In this process, a suspension with characteristic opalescence due to the small vesicles was obtained, which was filtered under sterile conditions by $0.2 \mu \mathrm{m}$. In the case of NC-EO-Aa, after the filtration step, a solution of $0.1 \mathrm{M} \mathrm{CaCl}_{2}$ was added [20]. In parallel, the same process was carried out to prepare NC without the EO-Aa, but containing the aqueous extract from S. saponaria. Finally, the preparations were stored in amber flasks at $4{ }^{\circ} \mathrm{C}$.

Following nanoformulation, the physicochemical characteristics of the nanocochleates were analyzed. The particle size (Ps) and polydispersion index (Pi) were carried out by Electrophoretic Light Scattering (ELS), using Delsa ${ }^{\mathrm{TM}}$-Nano C (Beckman Coulter, Stockholm, Sweden), with a detector of 165 degrees. Zeta (Z) Potential was also determined, taking into account the difference of particle motility induced by different magnetic fields. Data were processed by mathematic algorithms of Cumulant and Coutin. In each case, three replicates of each analysis were carried out and the results are expressed as mean \pm standard deviation.

\subsection{Parasites}

Leishmania amazonensis, strain MHOM/77BR/LTB0016, was generously donated by the Department of Immunology, Oswaldo Cruz Foundation (FIOCRUZ), Rio de Janeiro, Brazil, was used in this study. The parasites were regularly isolated from an infected BALB/c mouse and cultured in Schneider's medium (Sigma-Aldrich, St. Louis, MO, USA) containing 10\% heat-inactivated fetal bovine serum (HFBS, Sigma-Aldrich) and antibiotics (100 U of penicillin $/ \mathrm{mL}, 100 \mu \mathrm{g}$ of streptomycin/mL). Parasites were maintained as promastigotes at $26^{\circ} \mathrm{C}$ with passages every three or four days.

\subsection{Animals}

Protocols using laboratory animals were carried out according "Guideline on the Care and Use of Laboratory Animals", which was approved by the Ethics Committee from Institute of Tropical Medicine Pedro Kouri (CEI-IPK 14-12), Havana, Cuba. Forty female BALB/c mice with body masses of approximately 20 to $22 \mathrm{~g}$ were used. Animals were obtained from The National Centre of Laboratory Animals Production (CENPALAB), Havana, Cuba, and maintained under standard conditions.

\subsection{In Vitro Studies}

To evaluate the EO-Aa-NC, two experiments were performed: the antileishmanial activity against intracellular amastigotes of L. amazonensis [21] and cytotoxicity against peritoneal macrophage from $\mathrm{BALB} / \mathrm{c}$ mice [22]. In each case, a culture treated with NC or with EO-Aa, as well an untreated control, were also included. Briefly, peritoneal macrophages from normal BALB/c mice were obtained in RPMI medium (SIGMA, St. Louis, MO, USA) and antibiotics, seeded in 24-well Lab-Tek (Costar ${ }^{\circledR}$, San Diego, $\mathrm{CA}, \mathrm{USA}$ ) plates and incubated at $37^{\circ} \mathrm{C}$ and $5 \% \mathrm{CO}_{2}$. After $2 \mathrm{~h}$, free cells were removed and a culture of stationary-phase L. amazonensis promastigotes were added at a 4:1 parasite/macrophage ratio in the same medium supplemented with $10 \%$ HFBS. The plate was incubated again for $4 \mathrm{~h}$ under the same conditions and free parasites were removed. After that, $990 \mu \mathrm{L}$ of medium containing $10 \mu \mathrm{L}$ of the dissolved products were added in duplicate in the first well and serial dilutions 1:2 were performed. The plate was incubated for an additional $48 \mathrm{~h}$ under the same conditions. The supernatant was then discarded, the culture was washed, fixed with absolute methanol, stained with Giemsa, and examined 
under a light microscope with immersion oil. The infection rates were obtained by multiplying the percentage of infected macrophages by the number of amastigotes, counting in 25 macrophages per sample. Results are expressed as percent of reduction of the infection rate in comparison to those of the controls. For the cytotoxicity assay, peritoneal macrophages were also collected, seeded at $3 \times 10^{5}$ cells $/ \mathrm{mL}$ and incubated as described previously [23]. After $2 \mathrm{~h}$, the medium was removed and $50 \mu \mathrm{L}$ of medium with 10\% HFBS and antibiotics were added, with additional $48 \mu \mathrm{L}$ in the first wells and $2 \mu \mathrm{L}$ of each product. Serial dilutions (1:2) were then carried out and an additional $50 \mu \mathrm{L}$ of medium was added to each well. The plate was incubated at same conditions for $48 \mathrm{~h}$. Then, $15 \mu \mathrm{L}$ of 3-[4,5-dimethylthiazol-2-yl]- 2,5-diphenyltetrazolium bromide (MTT) (SIGMA, St. Louis, MO, USA) solutions ( $5 \mathrm{mg} / \mathrm{mL}$ in PBS, prepared and filtered at the moment of use) were added to each well and the plate was incubated under the same conditions. After $3 \mathrm{~h}$, the formazan crystals were dissolved with $100 \mu \mathrm{L}$ of dimethylsulfoxide (DMSO) and the optical density was measured at $560 \mathrm{~nm}$ and at $630 \mathrm{~nm}$ as a reference wavelength using a spectrophotometer (Sirio S Reader, 2.4-0, Seac and Radim Diagnostics, Calenzano, Italy). In each case, the $\mathrm{IC}_{50}$ was determined from lineal dose-response curves of three experiments. Results are expressed as means with respective standard deviations.

\subsection{In Vivo Studies}

Normal BALB/c mice were infected in the right hind footpad with $5 \times 10^{6}$ stationary-phase L. amazonensis promastigotes by subcutaneous route, designed as day 0 . After 4 weeks post-infection (p.i.), the mice were randomly divided into five groups of eight animals each using the random numbers table method and the treatment was initiated. Products (EO-Aa, EO-Aa-NC, NC and GTM) were administered at a dose of $30 \mathrm{mg} / \mathrm{kg}$ in a volume of $50 \mu \mathrm{L}$ by intralesional route every 4 days to complete 4 administrations. The additional group did not receive treatment. Lesion size was checked weekly by measuring footpad enlargement of the lesion diameter between 30 and 60 days p.i., using a digital calliper. Average lesion size was calculated as the differences obtained between infected and uninfected footpads. On day 45 and 60 p.i., three mice from each group were sacrificed by cervical dislocation and parasite burden determined by means of a culture microtitration technique as described Buffet and collaborators [24].

\subsection{Statistical Analysis}

Statistical differences, considered as $p<0.05$, between $\mathrm{IC}_{50}$ of the products were determined using the Mann-Whitney test; while lesion progression and parasite burden were analysed by the analysis of variance test, followed by a Post Hoc Test (LDS test or planned comparison). In all cases, Statistica for Windows Program (Release 4.5, StatSoft, Inc., Tulsa, OK, USA, 1993) was used.

\section{Results}

The purpose of the present study was to investigate the effect of essential oil from A. absinthium formulated in nanocochleate against $L$. amazonensis, including in vitro assays and in vivo models. In each assay, the antiparasitic activity and toxicity of nanocochleates of $A$. absinthium essential oil (EO-Aa-NC) were evaluated compared with the essential oil of A. absinthium itself (EO-Aa), as well as empty nanocochleates (NC).

\subsection{Preparation of Nanocochleates}

Nanocochleates of $A$. absinthium essential oil (EO-Aa-NC), as well as with the aqueous extract from S. saponaria (NC) were prepared by the dehydration-hydration process [17,18]. A tubular and enrolled structure was evident in these nanoformulations. The particle sizes (Ps) and polydispersity indices (Pi) of EO-Aa-NC and NC were below $100 \mathrm{~nm}$ and 0.5, respectively (Table 1), which are in concordance with polydispersion systems [25]. These results suggest that the formulations are heterogeneous suspensions. In addition, no volatile constituents, previously identify in the EO-Aa, 
were found in the supernatant of the formulations after analysis by gas-chromatography, indicating a high degree of formulation efficiency.

Table 1. Characteristics of nanocochleates.

\begin{tabular}{cccc}
\hline Formulation & Size $(\mathbf{n m}) \pm \mathbf{S D}$ & Polydispersity Index \pm SD & Z Potential (乙) $(\mathbf{m V}) \pm$ SD \\
\hline EO-Aa-NC & $74.2 \pm 21.9$ & $0.33 \pm 0.005$ & $-40.8 \pm 0.4$ \\
NC & $32.1 \pm 7.4$ & $0.48 \pm 0.04$ & $-31.2 \pm 0.08$ \\
\hline
\end{tabular}

EO-Aa-NC: essential oil from A. absinthium formulated in nanocochleates. NC: empty nanocochleates.

\subsection{In Vitro Antileishmanial Activity}

In vitro experiments were performed to evaluate the ability of the products to inhibit the proliferation of $L$. amazonensis amastigotes, as well as cytotoxicity against peritoneal macrophage from BALB/c mice (Table 2). In each case, the results are expressed in terms of median inhibitory concentration $\left(\mathrm{IC}_{50}\right)$. Regarding the inhibitory activity, EO-Aa-NC displayed lower inhibitory activity than EO-Aa $(p<0.05)$ on L. amazonensis-infected macrophages, which is probably due to the interval of time that the experiment was carry out. This effect could be a consequence of nanocochleate properties that acts as drug delivery systems of bioactive molecules [25].

Table 2. Antileishmanial activity and cytotoxicity effect of the essential oil from Artemisia absinthium L. collected in Havana, Cuba.

\begin{tabular}{|c|c|c|}
\hline \multirow{2}{*}{ Essential Oil from A. absinthium } & \multicolumn{2}{|c|}{$\mathrm{IC}_{50}{ }^{\mathrm{a}} \pm \mathrm{SD}^{\mathrm{b}}(\mu \mathrm{g} / \mathrm{mL})$} \\
\hline & Leishmania amazonensis & Peritoneal Macrophages \\
\hline EO-Aa & $13.4 \pm 2.4$ & $75.1 \pm 2.3$ \\
\hline EO-Aa-NC & $21.5 \pm 2.5 *$ & $27.7 \pm 5.6^{*}$ \\
\hline $\mathrm{NC}$ & c & $\mathrm{d}$ \\
\hline
\end{tabular}

${ }^{\mathrm{a}} \mathrm{IC}_{50}$ : concentration of product that caused $50 \%$ of inhibition growth. ${ }^{\mathrm{b}} \mathrm{SD}$ : standard deviation. EO-Aa: essential oil from A. absinthium. EO-Aa-NC: essential oil from A. absinthium formulated in nanocochleates. *: displays statistical differences $(p<0.05)$ compared to EO-Aa. NC: empty nanocochleates. ${ }^{\mathrm{c}} \mathrm{NC}$ inhibited $\sim 25 \%$ of intracellular amastigotes of L. amazonensis at maximum concentration tested. ${ }^{\mathrm{d}} \mathrm{NC}$ inhibited $\sim 70 \%$ of peritoneal macrophages at maximum concentration tested.

\subsection{In Vivo Antileishmanial Activity}

The EO-Aa-NC (30 mg/ kg/intralesional route/every four days four times) was tested in a murine model of CL, i.e., BALB/c mice infected with L. amazonensis in the footpad. Deaths and body weight were used as indicators of preliminary toxicity; while lesion thickness and parasite burden were measurements indicating disease progression. In addition, its antileishmanial efficacy was compared with animals treated with EO-Aa $(30 \mathrm{mg} / \mathrm{kg} /$ intralesional route/every four days four times) or NC (50 $\mu \mathrm{L}$ /intralesional route/every four days four times). Current antileishmanial chemotherapy GTM (30 mg/kg/intralesional route/every four days four times) was also included in the study. The lesion development in the infected animals was monitored for 10 weeks (see Figure 1). 


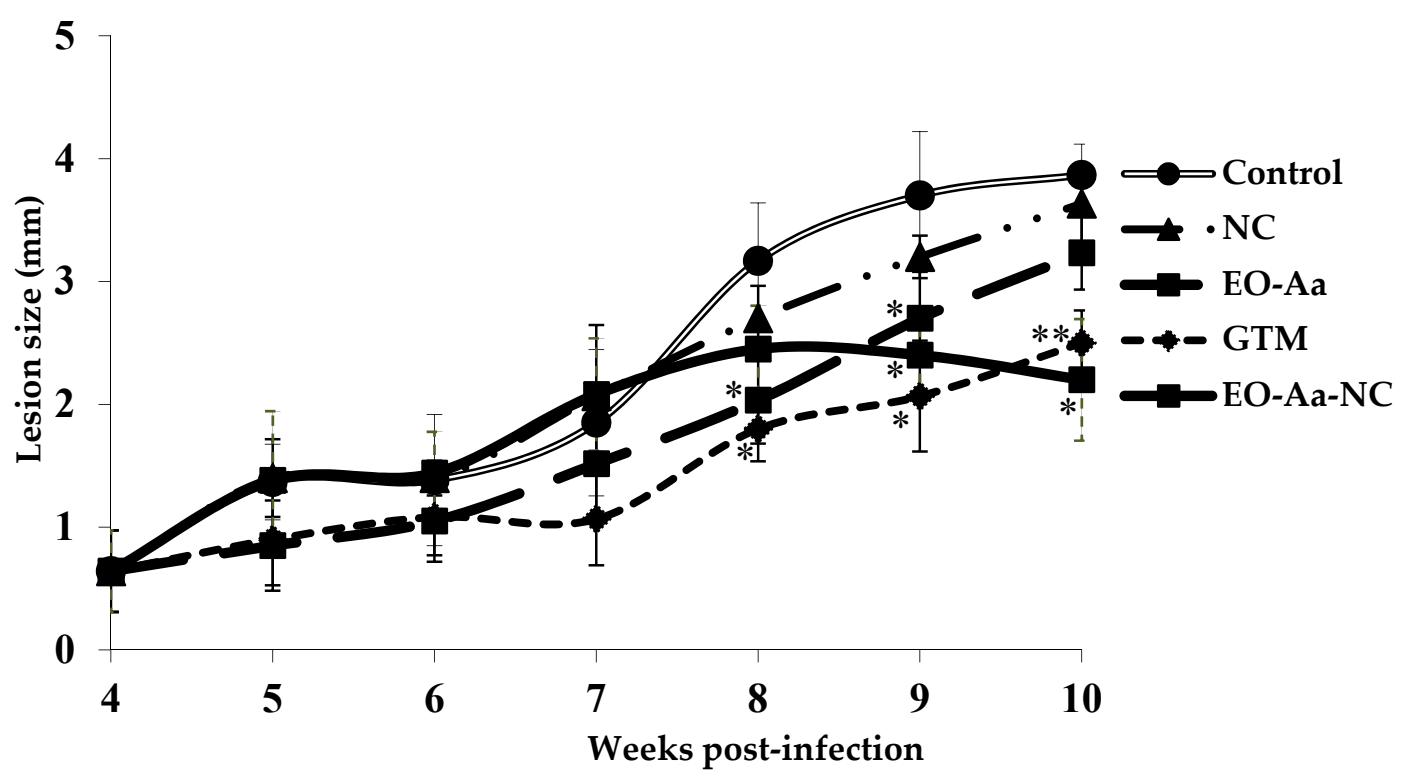

Figure 1. Effect of treatment with essential oil from Artemisia absinthium L. collected in Havana, Cuba, in comparison with the oil formulated in nanocochletaes. BALB/c mice were infected with L. amazonensis and 30 days post-infection the treatment was started with four doses by intralesional route at $30 \mathrm{mg} / \mathrm{kg}$ every four days. The results are expressed as mean of lesion size in infected area \pm standard deviation. EO-Aa: essential oil from A. absinthium; EO-Aa-NC: essential oil from A. absinthium formulated in nanocochleates; NC: empty nanocochleates; GTM: Glucantime ${ }^{\circledR}$ used as a reference drug. *: displays statistical differences $(p<0.05)$ compared to control untreated animals. ${ }^{* *}$ : displays statistical differences $(p<0.05)$ compared to animals treated with EO-Aa. a: a positive number represents an increase of body weight, while a negative number indicated decrease of body weight. EO-Aa: essential oil from A. absinthium. EO-Aa-NC: essential oil from A. absinthium formulated in nanocochleates. NC: empty nanocochleates. GTM: Glucantime ${ }^{\circledR}$ used as reference drug.

\section{Discussion}

\subsection{Preparation of Nanocochleates}

Current treatment options for leishmaniasis are generally considered to be less than satisfactory, mainly due to the high toxicity of the conventional drugs. There has been considerable attention recently given to plant-derived natural products in an attempt to discover new antileishmanial agents $[12,13]$. Recently, our research group reported the in vitro antileishmanial activity and in vivo efficacy of EO from A. absinthium [14]. However, it is known that EOs can easily decompose; a case where encapsulation represents a reasonable and efficient methodology to modulate drug release and increase the physical stability of the active substances. In addition, other properties could be improved including the increasing bioactivity, reduced toxicity, and improved patient compliance and convenience [26,27].

A significantly large fraction of the current literature on the encapsulation of essential oils deals with micrometric-sized capsules to decrease oil volatility and to transform the oil into a powder. Encapsulation in nanometric particles is an alternative for overcoming these complications but, additionally, due to the subcellular size, may also increase the cellular absorption with concomitant increase in bioefficacy $[15,26]$. Different drug delivery systems have been used to improve the therapeutic index and pharmacokinetics properties of antileishmanial drugs, including: liposomal formulations of amphotericin B [28], a mixed formulation of conventional and pegylated meglumine antimoniate-containing liposomes [29] and a nanoemulsion containing synthetic a chalcone [30]. In this sense, a novel lipid-based system are cochleate delivery vehicles, which are unique multilayered structures consisting of a large, continuous, solid lipid bilayer sheet rolled into a spiral, with no 
internal aqueous space [31]. However, the use of cochleates as alternatives in Leishmania treatment has been scarcely studied. In this sense, reports found in the literature include: (i) the encapsulation of amphotericin B into cochleates against L. chagasi [32] and (ii) the application of cochleates as adjuvants in a model of CL caused by L. major to promote a Th1 immune response [33].

In addition, Tamargo and collaborators have developed a technology to elaborate nanoliposomes and nanocochleates using phospholipids from soy (Glycine max L.) lecithin [19]. These nanoparticles have been evaluated as adjuvants and drug delivery systems of prophylactic or therapeutic antigens administered by systemic or mucosal routes in different experimental animal models. In this sense, all cases were demonstrated to induce humoral and cellular responses and protection, as well. Thus, nanoparticles not only constituted a new platform as an adjuvant that acts as a combined mechanism for a delivery system of antigen to promote the immune response, but also constituted a drug delivery system and carrier of different drugs and biomolecules [18,20].

\subsection{In Vitro Antileishmanial Activity}

The higher cytotoxicity $(p<0.05)$ on macrophages of EO-Aa-NC compared to EO-Aa is probably due to the influence of the nanocochleates. In addition, the same $\mathrm{IC}_{50}$ values $(p>0.05)$ of EO-Aa-NC were obtained between antileishmanial activity and cytotoxicity, corresponding to an unspecific activity. It may be that the high cytotoxicity observed is due to the presence of aqueous extracts from the pericarp of Sapindus saponaria L. (jaboncillo) plant, which is rich in saponins and was used in the formulation EO-Aa-NC [16]. The antileishmanial effects of this plant have been previously reported [34]; however, the specific cytotoxicity of these saponins is unknown, although in this study, lower quantities of extracts were used compared with the median hemolytic concentration $\left(\mathrm{HC}_{50}\right)$. Other toxicological and immunotoxicological studies of nanoliposomes and nanocochleates, developed using same technology used by Tamargo, demonstrated that this formulation without aqueous extracts from S. saponaria did not show cytotoxicity $[35,36]$.

\subsection{In Vivo Antileishmanial Activity}

With respect to efficacy evaluation, NC displayed the same lesion size $(p>0.05)$ compared to control animals through the course of the experiment, which demonstrated that empty nanocochleate formulation did not display antileishmanial activity. In contrast, the intralesional administration of the EO-Aa-NC was statistically superior $(p<0.05)$ than controls, suppressing infection in the murine model by approximately 50\% compared to untreated animals (Figure 1). In comparison to mice treated with EO-Aa, treatment with EO-Aa-NC also displayed smaller lesion size $(p<0.05)$. Finally, EO-Aa-NC was found to inhibit infection area growth as efficiently $(p>0.05)$ as administration of GTM. In this sense, Etel and collaborators also demonstrated that the encapsulated antileishmanial drugs are able to eliminate the amastigotes with higher efficiency than the free drugs [37].

Nevertheless, every group of animals that received treatment did not display differences $(p>0.05)$ with respect to parasite load ( $\log 10=3.33-4.20$ parasite $/ g)$. The progressive increase in the footpad lesions may have resulted from the inflammatory response to the infection itself; while decrease of thickness could be also be the result of anti-inflammatory effects induced by treatment. In addition, no complete cure was found in any group, which is the expected result in the murine model used. The inoculation of susceptible BALB/c mice with L. amazonensis promastigotes commonly results in massive and metastatic infection, transmission of the infection to visceral organs and consequently mouse death [38]. However, in the case of nanocochleate, as a controlled drug-delivery system, the active product is incorporated into a lipid network structure in such a way that the material is slowly released and in a predefined manner and as a consequence, the release time may be a few hours, days, up to several years $[39,40]$. This property could directly provide a better control of chronic diseases, as is the case of CL.

In this study, the intralesional route was used so that the product is completely deposited in the infected area, which would increase the contact between active compounds and Leishmania 
parasites and avoid potential metabolic degradation by the liver. Currently, the utility of intralesional administration has been demonstrated in clinically used drugs (Glucantime ${ }^{\circledR}[41]$ and Pentostam ${ }^{\circledR}$ [42]), as well as with natural products (Lippia sidoides [43] and Chenopodium ambrosioides [44]), including previous antileishmanial activity of EO-Aa [14]. Nevertheless, a systemic administration could prevent metastasis. In this sense, oral administration represents an interesting alternative and several studies of medicinal plants (Galipea longiflora [45], Kalanchoe pinnata [46] and Chenopodium ambrosioides [47]) have demonstrated this application. Although the EO-Aa has not been evaluated by oral route, the nanocochleates present several advantages, including: (i) they present a high percent of incorporation or association of molecules with different physicochemical characteristics (hydrophobic or hydrophilic), (ii) they are resistant to lyophilization processing without affecting the structure, (iii) and they can be administered by the oral route. In this sense, multiple applications have been proposed for nanoformulations as therapeutic agents, vaccines, or adjuvants [48,49].

\subsection{Future Directions}

As a promising strategy, potential antileishmanial drugs could include the combined effects of drug action and immunological status [50]. With this finding, in addition to the specific antileishmanial activity of EO-Aa-NC, the formulation may also enhance the immune response against the parasite. This is a desirable characteristic for a candidate drug for the treatment of CL. Therefore, this new formulation presents a high potential to explore as antileishmanial product. Further experiments aimed at the development of current nanoformulations are required, including toxicity, standardization of EO-Aa and stability of the formulation during the time and under the conditions present in the endemic countries of leishmaniasis.

\section{Conclusions}

In this study, EO-Aa was collected more than 15 years ago, which was stored under standard conditions with the aim to maintain consistent physical-chemical-biological properties of the oil. Therefore, the same sample previously reported was used for the present formulation [14]. However, in tropical countries where leishmaniasis is endemic, it is likely that standard storage conditions are very difficult to maintain due to high temperatures and severe economic problems that cause disruptions in electricity and failure to maintain cold temperatures. Thus, the search of new alternatives to protect the products and maintain their physical stability and activity is highly desirable. Therefore, in this study, encochleation of the EO from A. absinthium results in a stable, tolerable, and efficacious antileishmanial formulation, facilitating systemic delivery of the EO. This work demonstrates the in vivo activity of nanocochleate formulation when administered through the intralesional routes, which could be studied as an alternative treatment option for leishmaniasis and as a delivery system for EOs, increasing the activity of this preparation compared to administration of the free form EO.

Acknowledgments: This work was performed as part of the activities of the Research Network Natural Products against Neglected Diseases (ResNetNPND), http:/ / www.resnetnpnd.org/Start/.

Author Contributions: Beatriz Tamargo and Lianet Monzote designed the study. Beatriz Tamargo and Laura Machín prepared the nanocochleates; Abel Piñón, Lianet Monzote, and Marley García carried out the biological experiments. Ramón Scull extracted the essential oil and William N. Setzer carried out the chemical analysis. Beatriz Tamargo, Lianet Monzote and William N. Setzer wrote the manuscript. All authors reviewed the manuscript.

Conflicts of Interest: The authors declare no conflict of interest.

\section{References}

1. Schroeder, J.; Aebischer, T. Vaccine for leishmaniasis from proteome to vaccine candidates. Hum. Vaccins 2011, 7, 6. [CrossRef] 
2. Diniz, S.A.; Silva, F.L.; Carvalho, A.V.; Bueno, R.; Guerra, R.M.; Abreu-Silva, A.L.; Santos, R.L. Animal reservoirs for visceral leishmaniasis in densely populated urban areas. J. Infect. Dev. Ctries 2008, 2, 24-33. [PubMed]

3. Ashford, R.W. The leishmaniases as emerging and reemerging zoonoses. Int. J. Parasitol. 2000, 30, $1269-1281$. [CrossRef]

4. Alvar, J.; Vélez, I.D.; Bern, C.; Herrero, M.; Desjeux, P.; Cano, J.; Jannin, J.; denBoer, M.; The WHO Leishmaniasis Control Team. Leishmaniasis worldwide and global estimates of its incidence. PLoS ONE 2012, 7, e35671. [CrossRef] [PubMed]

5. De Paiva-Cavalcanti, M.; de Morais, R.K.S.; Pessoa-e-Silva, R.; Trajano-Silva, L.A.M.; Gonçalves-de-Albuquerque, S.C.; Tavares, D.H.C.; Brelaz-de-Castro, M.C.A.; Silva, R.F.; Pereira, V.R.A. Leishmaniases diagnosis: An update on the use of immunological and molecular tools. Cell Biosci. 2015, 5, 31. [CrossRef] [PubMed]

6. Kevric, I.; Cappel, M.A.; Keeling, J.H. New world and old world Leishmania infections a practical review. Dermatol. Clin. 2015, 33, 579-593. [CrossRef] [PubMed]

7. Schriefer, A.; Wilson, M.E.; Carvalho, E.M. Recent developments leading toward a paradigm switch in the diagnostic and therapeutic approach to human leishmaniasis. Curr. Opin. Infect. Dis. 2008, 21, 483-488. [CrossRef] [PubMed]

8. Mitropoulos, P.; Konidas, P.; Durkin-Konidas, M. New World cutaneous leishmaniasis: Updated review of current and future diagnosis and treatment. J. Am. Acad. Dermatol. 2010, 63, 309-322. [CrossRef] [PubMed]

9. Murray, H.W.; Berman, J.D.; Davies, C.R.; Saravia, N.G. Advances in leishmaniasis. Lancet 2005, 366, 1561-1577. [CrossRef]

10. Kedzierski, L.; Sakthianandeswaren, A.; Curtis, J.M.; Andrews, P.C.; Junk, P.C.; Kedzierska, K. Leishmaniasis: Current treatment and prospects for new drugs and vaccines. Curr. Med. Chem. 2009, 16, 599-614. [CrossRef] [PubMed]

11. Singh, N.; Kumar, M.; Singh, R.K. Leishmaniasis: Current status of available drugs and new potential drug targets. Asian Pac. J. Trop. Med. 2012, 5, 485-497. [CrossRef]

12. Fournet, A.; Muñoz, V. Natural products as trypanocidal, antileishmanial and antimalarial drugs. Curr. Top. Med. Chem. 2002, 2, 1215-1237. [CrossRef] [PubMed]

13. Tiuman, T.S.; Santos, A.O.; Ueda-Nakamura, T.; Filho, B.P.D.; Nakamura, C.V. Recent advances in leishmaniasis treatment. Int. J. Infect. Dis. 2011, 15, e525-e532. [CrossRef] [PubMed]

14. Monzote, L.; Piñón, A.; Scull, R.; Setzer, W.N. Chemistry and leishmanicidal activity of the essential oil from Artemisia absinthium from Cuba. Nat. Prod. Commun. 2014, 9, 1799-1804. [PubMed]

15. Bilia, A.R.; Guccione, C.; Isacchi, B.; Righeschi, C.; Firenzuoli, F.; Bergonzi, M.C. Essential oils loaded in nanosystems: A developing strategy for a successful therapeutic approach. eCAM 2014, 2014, 14. [CrossRef] [PubMed]

16. Mena, L.; Tamargo, B.; Salas, E.; Plaza, L.E.; Blanco, Y.; Otero, A.; Sierra, G.V. Determinación de saponinas y otros metabolitos secundarios en extractos acuosos de Sapindus saponaria L. (jaboncillo). Rev. Cuba Plantas Med. 2015, 20, 106-116.

17. Gregoriadis, G. Immunological adjuvants: A role for liposomes. Immunol. Today 1990, 11, 89-97. [CrossRef]

18. Tamargo, B.; Márquez, Y.; Ramírez, W.; Cedré, B.; Fresno, M.; Sierra, V.G. New proteoliposomic vaccine formulation from N. meningitidis serogroup B, without aluminum hydroxide, retains its antimeningococcal protectogenic potencial as well as Th-1 adjuvant capacity. BMC Immunol. 2013, 14 (Suppl. 1), S12. [CrossRef]

19. Tamargo, B.; Herrera, L.; Bello, A.; Cuéllar, A.; González, H.; Ortiz, L.; Morales, M.; Sierra, V.G. Obtención de fosfolípidos a partir de la Lecitina de Soya (Glicine max L.), para usos biomédicos. Rev. Cub. Quím. 2011, 23, 5-14.

20. Tamargo, B.; Rosario, L.A.; Batista, N.; Arencibia, D.F.; Fernández, K.; Villegas, A.; Ayala, J.A.; Sierra, V.G. Protección inducida por nanococleatos derivados de proteoliposomas de Leptospira interrogans serovar Canícola. VacciMonitor 2012, 21, 3-9.

21. Caio, T.S.E.; Lima, M.D.; Kaplan, M.A.C.; Nazareth, M.M.; Rossi-Bergmann, B. Selective effect of 2',6'dihydroxy-4'methoxychalcone isolated from Piper aduncum on Leishmania amazonensis. Antimicrob. Agents Chemother. 1999, 43, 1234-1241.

22. Sladowski, D.; Steer, S.J.; Clothier, R.H.; Balls, M. An improved MTT assay. J. Immunol. Method 1993, 157, 203-207. [CrossRef] 
23. Monzote, L.; Piñón, A.; Setzer, W.N. Antileishmanial potential of tropical rainforest plant extracts. Medicines 2014, 1, 32-55. [CrossRef]

24. Buffet, P.A.; Sulahian, A.; Garin, Y.J.F.; Nassar, N.; Derouin, F. Culture microtitration a sensitive method for quantifying Leishmania infantum in tissues of infected mice. Antimicrob. Agents Chemother. 1995, 39, 2167-2168. [CrossRef] [PubMed]

25. Pawar, A.; Bothiraja, C.; Shaikh, K.; Mali, A. An insight into cochleates, a potential drug delivery system. RSC Adv. 2015, 5, 81188-81202. [CrossRef]

26. Ravi, K.M.N. Nano and microparticles as controlled drug delivery devices. J. Pharm. Pharm. Sci. 2000, 3, 234-258.

27. Turek, C.; Stintzing, F.C. Stability of essential oils: A review. Compr. Rev. Food Sci. Food Saf. 2013, 12, 40-53. [CrossRef]

28. Manandhar, K.D.; Yadav, T.P.; Prajapati, V.K. Antileishmanial activity of nano-amphotericin B deoxycholate. J. Antimicrob. Chemother. 2008, 62, 376-380. [CrossRef] [PubMed]

29. Azevedo, E.G.; Ribeiro, R.R.; da Silva, S.M.; Ferreira, C.S.; de Souza, L.E.; Ferreira, A.A.; de Oliveira, E.; Castro, R.A.; Demicheli, C.; Rezende, S.A.; et al. Mixed formulation of conventional and pegylated liposomes as a novel drug delivery strategy for improved treatment of visceral leishmaniasis. Expert Opin. Drug Deliv. 2014, 11, 1551-1560. [CrossRef] [PubMed]

30. Mattos, C.B.; Argenta, D.F.; Melchiades, G.L.; Cordeiro, M.N.S.; Tonini, M.L.; Moraes, M.H.; Weber, T.B.; Roman, S.; Nunes, R.J.; Teixeira, H.F.; et al. Nanoemulsions containing a synthetic chalcone as an alternative for treating cutaneous leshmaniasis: Optimization using a full factorial design. Int. J. Nanomed. 2015, 10, 5529-5542.

31. Santangelo, R.; Paderu, P.; Delmas, G.; Chen, Z.W.; Mannino, R.; Zarif, L.; Perlin, D.S. Efficacy of oral cochleate-amphotericin B in a mouse model of systemic candidiasis. Antimicrob. Agents Chemother. 2000, 44, 2356-2360. [CrossRef] [PubMed]

32. Sesana, A.M.; Monti-Rocha, R.; Vinhas, S.A.; Morais, C.G.; Dietze, R.; Lemos, E.M. In vitro activity of amphotericin B cochleates against Leishmania chagasi. Mem. Inst. Oswaldo Cruz 2011, 106, 251-253. [CrossRef] [PubMed]

33. Pérez, O.; Bracho, G.; Lastre, M.; Mora, N.; Del Campo, J.; Gil, D.; Zayas, C.; Acevedo, R.; González, D.; López, JA.; et al. Novel adjuvant based on a proteoliposome-derived cochleate structure containing native lipopolysaccharide as a pathogen-associated molecular pattern. Immunol. Cell Biol. 2004, 82, 603-610. [CrossRef] [PubMed]

34. Zarif, L. Drug delivery by lipid cochleates. Methods Enzymol. 2005, 391, 314-329. [PubMed]

35. Tamargo, B.; Sierra, V.G.; Fleitas, C.; Infante, J.F.; Ramírez, W.; Marquéz, Y.; Pérez, V.; Torralba, D.; García, L.; Burg, V.; et al. Evaluación inmunotoxicológica, de nuevas formulaciones adyuvantes nanoparticulados sin $\mathrm{Al}(\mathrm{OH})_{3}$, ensayadas como candidatos vacunales contra Neisseria meningitidis. VacciMonitor 2011, 20, 10.

36. Rodríguez-Hernández, D.; Barbosa, L.C.; Demuner, A.J.; de Almeida, R.M.; Fujiwara, R.T.; Ferreira, S.R. Highly potent anti-leishmanial derivatives of hederagenin, a triperpenoid from Sapindus saponaria L. Eur. J. Med. Chem. 2016, 124, 153-159. [CrossRef] [PubMed]

37. Etel, S.; Borborema, T. Uptake antileishmanial activity of meglumine antomoniate-containing liposomes in Leishmania (Leishmania) major- infect macrofages. Int. J. Antimicrob. Agents 2011, 38, 341-347.

38. Rafiee, A.; Riazi-rad, F.; Darabi, H.; Khaze, V.; Javadian, S.; Ajdary, S.; Bahrami, F.; Alimohammadian, A.H. Ferroportin-encapsulated nanoparticles reduce infection and improve immunity in mice infected with Leishmania major. Int. J. Pharm. 2014, 466, 375-381. [CrossRef] [PubMed]

39. Darole, P.S.; Hegde, D.D.; Nair, H.A. Formulation and evaluation of microemulsion based delivery system for amphotericin B. AAPS Pharm. Sci. Tech. 2008, 9, 122-128. [CrossRef] [PubMed]

40. Jogani, V.; Jinturkar, K.; Vyas, T.; Misra, A. Recent patents review on intranasal administration for CNS drug delivery. Recent Pat. Drug Deliv. Formul. 2008, 2, 25-40. [PubMed]

41. Salmanpour, R.; Razmavar, M.R.; Abtahi, N. Comparison of intralesional meglumine antimoniate, cryotherapy and their combination in the treatment of cutaneous leishmaniasis. Int. J. Dermatol. 2006, 45, 1115-1116. [CrossRef] [PubMed] 
42. El-Sayed, M.; Anwar, A.E. Intralesional sodium stibogluconate alone or its combination with either intramuscular sodium stibogluconate or oral ketoconazole in the treatment of localized cutaneous leishmaniasis: A comparative study. J. Eur. Acad. Dermatol. Venereol. 2010, 24, 335-340. [CrossRef] [PubMed]

43. De Medeiros, M.D.; da Silva, A.C.; Citó, A.M.; Borges, A.R.; de Lima, S.G.; Lopes, J.A.; Figueiredo, R.C. In vitro antileishmanial activity and cytotoxicity of essential oil from Lippia sidoides Cham. Parasitol. Int. 2011, 60, 237-241. [CrossRef] [PubMed]

44. Monzote, L.; Pastor, J.; Scull, R.; Gille, L. Antileishmanial activity of essential oil from Chenopodium ambrosioides and its main components against experimental cutaneous leishmaniasis in BALB/c mice. Phytomedicine 2014, 21, 1048-1052. [CrossRef] [PubMed]

45. Fournet, A.; Ferreira, M.E.; Rojas de Arias, A.; Torres de Ortiz, S.; Fuentes, S.; Nakayama, H. In vivo efficacy of oral and intralesional administration of 2-substituted quinolines in experimental treatment of New World cutaneous leishmaniasis caused by Leishmania amazonensis. Antimicrob. Agents Chemother. 1996, 40, 2447-2451. [PubMed]

46. Da Silva, S.A.G.; Costa, S.S.; Mendoça, S.C.F.; Silva, E.M.; Moraes, V.L.G.; Rossi-Bergmann, B. Therapeutic effect of oral Kalanchoe pinnata leaf extract in murine leishmaniasis. Acta Trop. 1995, 60, 201-210. [CrossRef]

47. Monzote, L.; García, M.; Montalvo, A.M.; Linares, R.; Scull, R. Effect of oral treatment with the essential oil from Chenopodium ambrosioides against cutaneous leishmaniasis in BALB/c mice, caused by Leishmania amazonensis. Res. Complement. 2009, 16, 334-338. [CrossRef] [PubMed]

48. Gould-Fogerite, S.; Kheiri, M.T.; Zhang, F.; Wang, Z.; Scolpino, A.J.; Feketeova, E; Canki, M.; Mannino, R.J. Targeting immune response induction with cochleate and liposome-based vaccines. Adv. Drug Deliv. Rev. 1998, 32, 273-287. [CrossRef]

49. Gould-Fogerite, S.; Mannino, R.J. Mucosal and Systemic Immunization using Cochleate and Liposome Vaccine. J. Liposome Res. 1996, 6, 357-379. [CrossRef]

50. Sacks, D.L.; Lal, S.L.; Shrivastava, S.N.; Blackwell, J.; Neva, F.A. An analysis of T-cell responsiveness in Indian kala-azar. J. Immunol. 1987, 138, 908-913. [PubMed]

(C) 2017 by the authors. Licensee MDPI, Basel, Switzerland. This article is an open access article distributed under the terms and conditions of the Creative Commons Attribution (CC BY) license (http:/ / creativecommons.org/licenses/by/4.0/). 